\title{
Single-shot phase and amplitude fluctuations of narrow-line pulse bursts in divided-pulse amplifier
}

\author{
Huaiqin Lin, Yujun Feng, Jonathan H. V. Price, and Johan Nilsson
}

\begin{abstract}
We demonstrate single-shot temporal-based interferometric phase measurements for bursts of 35 pulses of 1ns duration in an Yb-doped fiber amplifier chain seeded by a 1063$\mathrm{nm}$ single-frequency diode laser and operating in a highly nonlinear and saturated regime. We determined a maximum intra-pulse phase distortion ( $B$-integral) of $21.2 \mathrm{rad}$ by mixing the burst with a reference. The difference in the $B$-integral between the first and last pulse in the burst was large, $12.8 \mathrm{rad}$, but can be compensated for. Moreover, burst-to-burst amplitude fluctuations (standard deviation, std) were below $6 \%$, where phase fluctuations were $\sim 0.6 \mathrm{rad}$ (std) or less. Even if these fluctuations cannot be compensated for, they still allow for efficient coherent combination, which opens up for transform-limited or narrow-line high-energy divided-pulse amplification with single-frequency seeding.
\end{abstract}

Index Terms - Optical fiber lasers, optical fiber amplifiers, phase measurement

\section{INTRODUCTION}

$\mathrm{D}$ vided-pulse amplification (DPA) of a burst of optical pulses [1] followed by temporal coherent recombination (stacking) into a single pulse is a recently emerged option for pulse amplification to high peak power and energy [2], [3]. Also known as coherent pulse stacking amplification (CPSA), it is typically used for femtosecond pulses [4]. Combined with chirped-pulse amplification (CPA), the pulses can be stretched to $\sim 1 \mathrm{~ns}$ and then further effective stretching by DPA avoids excessive nonlinearities and damage even at high energy extraction [5], with burst energy several times the saturation energy. CPSA works with other types of coherent pulses, too.

Amplitude and phase distortions reduce the stacking efficiency of CPSA [4]. Thus, the achievable efficiency can be degraded by gain saturation in the amplifier and any mismatch of the accumulated phase between pulses due to peak-powerrelated self-phase modulation (SPM) [6]. Higher peak power is more demanding in that a given relative change results in a larger change of the $B$-integral (which quantifies SPM), and thus in larger stacking-efficiency degradation [7]. Similarly, phase distortions caused by the Kramers-Kronig (K-K) effect [8] and instantaneous heating [9] resulting from the quantum defect or photodarkening increase with higher pulse energy. To understand the potential of CPSA, it is necessary to characterize

This work was supported by the Air Force Office of Scientific Research under Grant FA9550-14-1-0382. (Corresponding author: Yujun Feng.) amplitude and phase distortions within the amplified burst, and estimate their stability, as affected by gain saturation and nonlinearities in parameter regimes of interest. The intra-pulse nonlinear chirp profile of single pulses has been measured via a best fit for $B$-integrals up to $38 \mathrm{rad}$ [10]. For bursts, we have previously measured the phase of individual pulses for a single amplifier [11]. However, this was for small $B$-integral (2.4 rad) and burst energy $(10 \mu \mathrm{J} v s .25 \mu \mathrm{J}$ saturation energy).

In this paper, we demonstrate DPA in an Yb-doped fiber amplifier (YDFA) chain in the regime of transform-limited seed pulses, formed by a single-frequency diode laser externally modulated to bursts of 35 1-ns Gaussian-like pulses ( $\sim 0.44 \mathrm{GHz}$ transform-limited bandwidth). We characterize the amplitude and phase at high extracted energy with a single-shot homodyne method, which we describe. Given a measurement bandwidth of $\sim 12.5 \mathrm{GHz}$, the method can handle such pulses with $B \leq 50 \mathrm{rad}$. The maximum extracted energy was $30.7 \mu \mathrm{J}$ (vs. $25 \mu \mathrm{J}$ saturation energy) and the maximum phase distortion was $21.2 \mathrm{rad}$. The $B$-integral difference between the burst's first and last pulse was then $12.8 \mathrm{rad}$. The burst-to-burst fluctuation of individual pulses was measured to $<6 \%$ (std) in instantaneous power and $0.6 \mathrm{rad}$ (std) in phase. This is still within suggested phase tolerances $(\lambda / 10)$ for stacking. We do not stack the amplified pulses, but note that $0.6 \mathrm{rad}$ (std) phase fluctuations allow for $91 \%$ of maximum stacking efficiency [12].

\section{METHOD}

Figure 1 shows our Yb-doped fiber (YDF) DPA and measurement system. All fibers and fiber components are polarization-maintaining (PM) for the signal and reference, which were polarized throughout. The seed was a single-frequency continuous-wave (CW) distributedfeedback (DFB) diode laser (II-VI CMDFB1064A, driven by a Newport Model 560 laser diode driver) with $\sim 1 \mathrm{MHz}$ linewidth and protected by an isolator. A fiber coupler then tapped off half of the power to serve as $\mathrm{CW}$ reference. The other half was passed through an electro-optic modulator (EOM, EOSpace AZ-2K5-10-PFU-PFU108), driven by an amplified $12 \mathrm{GSa} / \mathrm{s}$ arbitrary waveform generator (Tektronix AWG7122B) to generate 1-MHz bursts of 35 1-ns pulses with $10 \mathrm{~ns}$ period. A bias controller (YY Labs Mini-MBC-1) ensured adequate extinction ratio. The bursts were then pre-amplified by two YDFAs, each with $7.5 \mathrm{~m}$ of 5- $\mu \mathrm{m}$-core fiber (Nufern PM-YDF-5/130-

The authors are with the Optoelectronics Research Centre, University of Southampton, Southampton SO17 1BJ, U.K. (e-mail: H.Q.Lin@soton.ac.uk; Yujun.Feng@soton.ac.uk; jhvp@orc.soton.ac.uk; jn@orc.soton.ac.uk). 


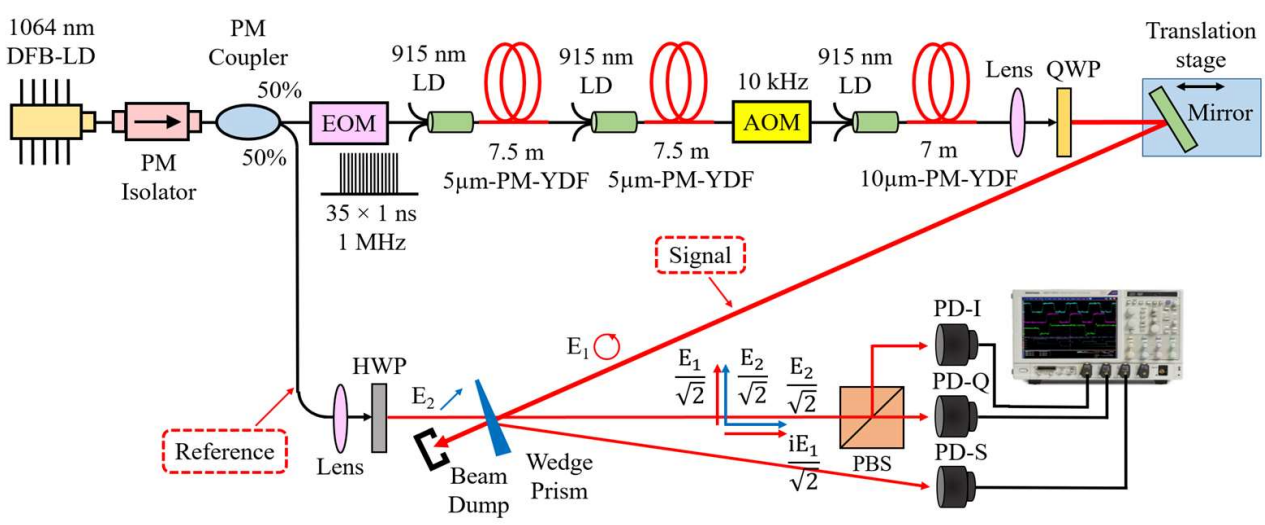

Fig. 1. Schematic of the pulse-burst fiber amplifier and measurement system. Pump diodes: JDSU 6397-L3 Series diode laser (915 nm), Pump diode drivers: Laser Driver Inc. Model LDI-820 (1st amplifier), SDL-822 (2nd amplifier), TDK-Lambda, GEN40-38 (3rd amplifier).

VIII). Then, an acousto-optic modulator (AOM, Gooch \& Housego T-M200-0.1C2G-3-F2P with driver Gooch \& Housego 1200AFDINA-2.5 HCR) optionally reduced the burst repetition frequency to $10 \mathrm{kHz}$ for high energy extraction. Here, the bursts had energy of $0.13 \mu \mathrm{J}$, temporal contrast of $36 \mathrm{~dB}$, and $\sim 1 \%$ (std) fluctuations in the peak power of the leading pulse. Finally, the bursts were amplified by a 10- $\mu \mathrm{m}$-core YDFA comprising $7 \mathrm{~m}$ of Nufern PLMA-YDF-10/125VIII. At the output of each amplifier, a polarizing isolator with built-in bandpass filter removed amplified spontaneous emission and blocked backward-propagating light.

To retrieve the temporal phase, we measured the instantaneous power of the burst signal $I_{1}(t)=\left|E_{1}(t)\right|^{2}$ and, following interference with the $\mathrm{CW}$ reference $I_{2}=\left|E_{2}\right|^{2}$, its in-phase $I_{I}(t)$ and quadrature $\left(90^{\circ}\right.$-shifted) $I_{Q}(t)$ homodyne signals via single-shot highspeed detection. Compared to the pulsed reference we used previously [11], a CW reference removes the need for pulse synchronization. Experimentally, we set up a $90^{\circ}$ optical hybrid and generated the signals according to Fig. 1. The output beam from the final amplifier was collimated and passed through a quarter-wave plate (QWP) placed with principal axes at $45^{\circ}$ to the linear polarization of the output beam. The resulting circular polarization is here best thought of as two linearly polarized components with equal strength and $90^{\circ}$ phase difference. A glass wedge at a small angle-of-incidence then recombined the output beam with the collimated reference beam. The reference is linearly polarized at $45^{\circ}$ to the QWP's principal axes, so the combined beam carries both $I_{I}(t)$ and $I_{Q}(t)$ homodyne signals. These were split by a polarization beam splitter aligned with the principal axes and then detected along with $I_{1}$ (as reflected in the wedge) by biased photodiodes PD-I, PD-Q, and PD-S (EO Technics ET-3500, bandwidth $>12.5 \mathrm{GHz}$ ). A multi-channel, 20-GHz oscilloscope (Tektronix DSA72004B, $50 \mathrm{GSa} / \mathrm{s}$ sampling in each channel simultaneously) then acquires single-shot traces from these three photodiodes. We get [13],

$$
\begin{gathered}
I_{I}(t)=\left|\frac{E_{1}(t)}{\sqrt{2}}+\frac{E_{2}}{\sqrt{2}}\right|^{2}=\frac{I_{1}(t)}{2}+\frac{I_{2}}{2}+\left[I_{1}(t) I_{2}\right]^{1 / 2} \cos [\varphi(t)] \\
I_{Q}(t)=\left|\frac{i E_{1}(t)}{\sqrt{2}}+\frac{E_{2}}{\sqrt{2}}\right|^{2}=\frac{I_{1}(t)}{2}+\frac{I_{2}}{2}+\left[I_{1}(t) I_{2}\right]^{1 / 2} \sin [\varphi(t)] \\
\varphi(t)=\tan ^{-1}\left\{\frac{I_{Q}(t)-I_{1}(t) / 2-I_{2} / 2}{I_{I}(t)-I_{1}(t) / 2-I_{2} / 2}\right\}
\end{gathered}
$$

Here, $\varphi(t)$ is the sought-after temporal phase of $E_{1}(t)$, i.e., of the signal burst, which was finally calculated in Matlab. In all cases, presented results are based on data from 100 bursts which were acquired within $30 \mathrm{~s}$ and averaged and used for fluctuation analysis. Although a slow drift could be significant over $30 \mathrm{~s}$, we verified that the fluctuations were similar on a burst-to-burst basis. We did not evaluate errors directly, but our measurements suggest that our system can keep random errors within $0.2 \mathrm{rad}$ in regions of adequate power. In a similar setup, we were able to measure fluctuations of $0.1 \mathrm{rad}$ (std) at low B-integral [11]. Simulations showed that the maximum $B$ integral for which the phase profile could be retrieved, with $12.5 \mathrm{GHz}$ bandwidth and 1-ns Gaussian pulses, was $\sim 50 \mathrm{rad}$ under idea conditions. Noise, jitter and pulse irregularities may reduce this value.

\section{RESULTS}

The instantaneous power profiles are shown in Fig. 2. We used two different pump powers for the final YDFA, resulting in burst output energies of $11.5 \mu \mathrm{J}$ and $30.7 \mu \mathrm{J}$, which can be compared to the saturation energy of $25 \mu \mathrm{J}$ calculated from the fiber parameters. In Fig. 2(a), both bursts show a characteristic quasi-exponential decay of the power envelope, caused by gain compression as energy is extracted from the amplifier. The first and last pulse within the bursts are shown in Fig. 2(b) with an enlarged and shifted time scale. Due to different amounts of gain saturation, the amplitudes of the first pulse are 2.85 and 1.67 times as high as for the last pulse for the high-power (HP) and low-power (LP) outputs, respectively. For comparison, the gain compression values expected from the extracted energy become 3.36 and 1.57 , respectively.

The change in peak power within a burst can be problematic, but the seed can be pre-compensated to equalize the peak output power or the $B$-integral [14]. CPSA also works with varying pulse peak power within the burst $[4,15]$. However, there are also fast burst-to-burst fluctuations in how the pulse amplitude evolves within a burst. These cannot be pre-compensated. Figure 3 shows the std in the peak power of each of the 35 pulses, evaluated for 100 bursts. The std varies in a similar way as the peaks in Fig. 2 (a), and is between $4.5 \%$ and $6 \%$ of the peaks in the LP case and between $3.5 \%$ and $4.5 \%$ of the peaks in the HP case. The std remains similar over the central $0.5 \mathrm{~ns}$ of the pulses and are considerably larger than the $1 \%$ (std) pulse 
peak fluctuation at the input to the final YDFA.

The energy gain grows exponentially with stored energy in the unsaturated regime, but approximately linearly when saturated. This may explain why the relative fluctuations are smaller at high power. A std in the instantaneous power of $6 \%$ degrades the stacking efficiency by $0.09 \%$ in case of 35 pulses. For more pulses the degradation is smaller [16].
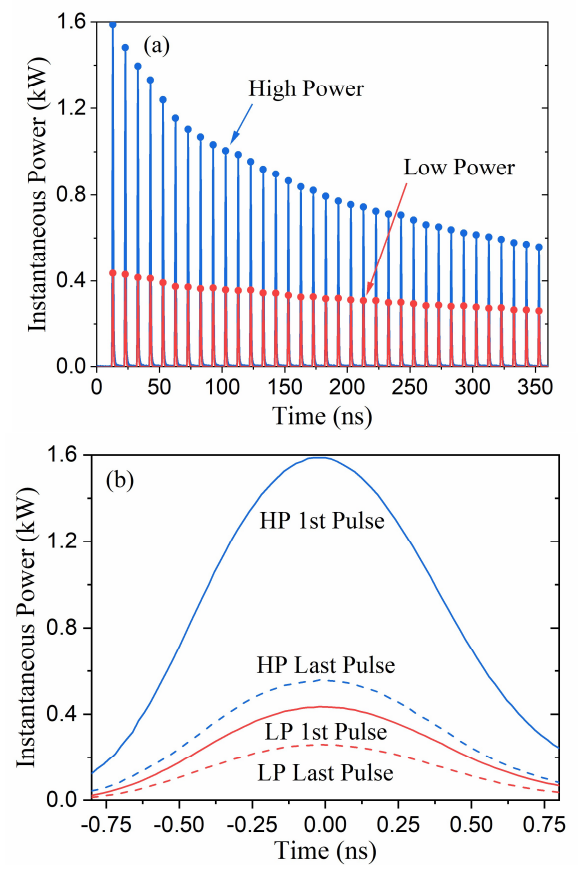

Fig. 2. Instantaneous output power averaged over 100 bursts for high and low power (a) for the whole burst and (b) the first and last pulse.

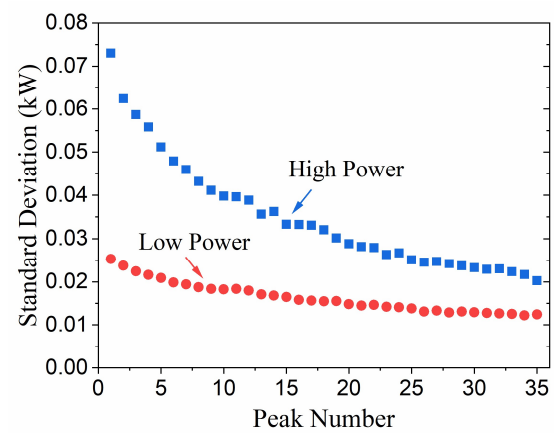

Fig. 3. Std in peak power calculated for 100 bursts for each of the 35 pulses.

There will also be variations in the phase, e.g., because of SPM, K-K and thermal effects. Figure 4 shows the instantaneous phase calculated according to Eq. (3), for the whole burst and enlarged for the first and last pulses. Pulse stacking does not depend on the absolute phase, so we have added a constant to make the peak phase of the first pulse in the burst $0 \mathrm{rad}$. The phase was then evaluated for the range of each pulse where the instantaneous power was large enough to yield meaningful results. Typically, this was over the central $1.6 \mathrm{~ns}$. The phase varies over several waves, and was therefore unwrapped. The procedure was then repeated for the next pulse. Since the instantaneous phase is not available continuously between pulses, we adjusted the peak phase by integer factors of $2 \pi$ to make the change from the previous peak the smallest possible. This was in all cases negative with magnitude $<1 \mathrm{rad}$.

Within a pulse, Fig. 4(b) shows that for the first pulse, the phase varies by $10.6 \mathrm{rad}$ (HP) and by $3.3 \mathrm{rad}$ (LP) within the central $1 \mathrm{~ns}$. The variations in the last pulse are only $4.4 \mathrm{rad}$ and $2.2 \mathrm{rad}$. The difference in phase profile between the first and last pulse is in both cases much larger than the acceptable $\lambda / 10$, so the instantaneous phase would have to be corrected across the pulses for efficient combination. The phase profiles are similar to the instantaneous-power profiles, which suggests that SPM dominates the phase distortion. This was also seen in simulations in Ref. [9]. The full variations in phase, if dominated by SPM, are expected to be approximately twice as large as the variations over the FWHM duration, since those only correspond to power variations from $50 \%$ to $100 \%$ of the peak power. Thus, for the whole pulse, the phase variations of the first pulse may reach $21.2 \mathrm{rad}$ (HP) and $6.6 \mathrm{rad}$ (LP). These values are approximately equal to the $B$-integral and are well within the estimated 50-rad limit. The first HP pulse has a maximum deviation of the instantaneous frequency of $5.3 \mathrm{GHz}$ and a linewidth of $10 \mathrm{GHz}$ (23 times the transform limit) as calculated from the measured phase $\varphi(t)$. According to calculations of the pulse evolution through the amplifiers. the peak power of the first pulse becomes $1.6 \mathrm{~kW}(0.4 \mathrm{~kW})$ in the HP (LP) case, and the B-integral becomes $18.6 \mathrm{rad}$ (4.4 rad) in the main amplifier and $20.8 \mathrm{rad}(6.4 \mathrm{rad})$ in the whole amplifier chain. Thus, the measured phase differences are in fair agreement with the $B$-integral calculations. Besides, the ratios of the measured intra-pulse phase variations between the first and the last pulse are 2.5 (HP) and 1.5 (LP), which is also matched to the aforementioned ratios in peak power.
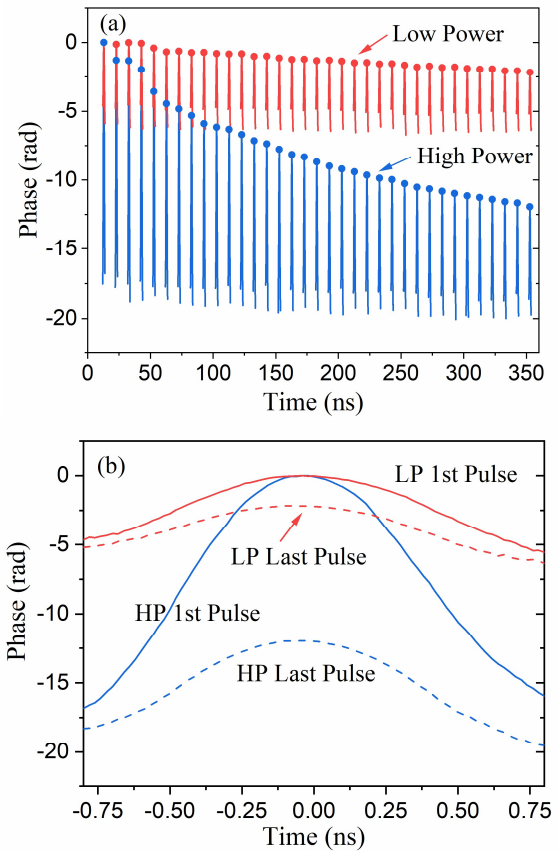

Fig. 4. (a) High- and low-power phase profiles for (a) bursts and (b) the first and last pulses. The curves are averaged over 100 bursts.

However, the phase in the trailing edge of the pulses is smaller than that in the leading edge, by an amount of between 0.6 and $1.3 \mathrm{rad}$ at $0.5 \mathrm{~ns}$. This asymmetry may be caused by 
energy-related phase effects, which build up during the pulse [9]. In contrast to these experiments, the simulations in [9] showed larger phase in the trailing edge than in the leading edge. This suggests that the refractive index decreases through the combined effect of energy extraction from the $\mathrm{Yb}$-ions and heat deposition. The asymmetry corresponds to a value of Henry's $\alpha$-parameter [17] of $\sim 60$. There is also a suggestion that the asymmetry is stronger towards the end of the burst, which indicates that the time constant for at least a part of this energydependent phase effect is slower than the pulse separation of $10 \mathrm{~ns}$. The phase asymmetry of $1.3 \mathrm{rad}$ may already be significant, and is furthermore expected to increase for higher extracted energy per pulse. Note also that in contrast to SPM, this phase distortion is not expected to become smaller in shorter fibers.

CPSA requires that burst-to-burst variations in the instantaneous phase are similar for each pulse (simplest is that they are small). Figure 5 shows the std of the relative phase for the last pulse, which is defined as the phase difference between the last and the first pulse within a burst. The std is below $0.6 \mathrm{rad}(\mathrm{HP})$ and $(0.3 \mathrm{rad})(\mathrm{LP})$ at the peaks at $0 \mathrm{~ns}$, and nearly constant over the central $1 \mathrm{~ns}$ of the pulses. The std is larger in the pulse edges, but there, the energy and instantaneous power are small, and there may well be larger measurement errors caused by noise. The same analysis of the instantaneous phase of the central parts of other pulses, relative to that of the first pulse, gave similar std-values. This std appeared to increase towards higher pulse numbers, but the trend was not clear.

Whereas the average effects in Fig. 2(a) and Fig. 4(a) are possible to compensate for with amplitude and phase controllers, the fluctuations (std) in Fig. 3 and Fig. 5 are more difficult to handle. However, the $0.6 \mathrm{rad}$ (std) of fluctuation still allows for $91 \%$ of stacking efficiency [12].

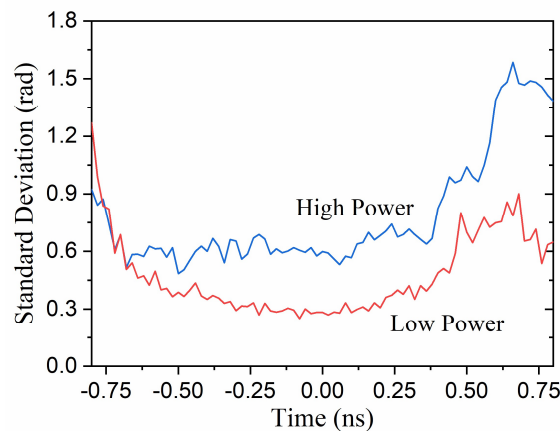

Fig. 5. Std of the instantaneous phase of the last pulse within the burst relative to that of the first pulse at high and low power.

\section{CONCLUSION}

We have demonstrated single-shot high-speed phase measurements of spectrally narrow pulses. The maximum phase distortion reached 21.2 rad in a burst of 35 1-ns Gaussianlike pulses at $1063 \mathrm{~nm}$ from an Yb-doped fiber amplifier chain through mixing with a continuous-wave reference. In this highly nonlinear saturated regime, the differences in phase profile between pulses within a burst were so large that the intra-pulse phase profile would need to be corrected if the pulses are to be temporally recombined (stacked) into a single pulse. In addition, $6 \%$ (std) amplitude fluctuation and $0.6 \mathrm{rad}$ (std) (i.e., $\lambda / 10$ ) phase fluctuation between bursts were also diagnosed. The fluctuations arise primarily in the final YDFA. They cannot be corrected, but are not so large that they preclude efficient pulse stacking. Simulations showed that with the used pulse shape and duration, a measurement bandwidth of $\sim 12.5 \mathrm{GHz}$ allows for $B$-integral up to $\sim 50 \mathrm{rad}$, which exceeds the value expected in CPSA systems. We note that our results are also relevant for chirped- and divided-pulse amplification (CDPA) systems for fs pulses. These can also be directly characterized with a similar approach by the use of a chirpedpulse reference pathlength-matched to within $1 \mathrm{~mm}$ to avoid excessive beat frequencies.

\section{ACKNOWLEDGMENT}

The authors thank James R. Leger and W. Minster Kunkel, University of Minnesota, for fruitful discussions. H. Lin acknowledges financial support by the China Scholarship Council. All supporting data are available from the University of Southampton at http://doi.org/10.5258/SOTON/D0600.

\section{REFERENCES}

[1] S. Zhou, F. W. Wise, and D. G. Ouzounov, "Divided-pulse amplification of ultrashort pulses," Opt. Lett., vol. 32, no. 7, pp. 871-873, Apr. 2007.

[2] Y. Zaouter et al., "Femtosecond fiber chirped- and divided-pulse amplification system," Opt. Lett., vol. 38, no. 2, pp. 106-108, Jan. 2013.

[3] M. Kienel, et al., "Energy scaling of femtosecond amplifiers using actively controlled divided-pulse amplification," Opt. Lett., vol. 39, no. 4, pp. 1049-1052, Feb. 2014.

[4] T. Zhou, J. Ruppe, C. Zhu, I. Hu, J. Nees, and A. Galvanauskas, "Coherent pulse stacking amplification using low-finesse Gires-Tournois interferometers," Opt. Express, vol. 23, no. 6, pp. 7442-7462, Mar. 2015.

[5] F. Guichard, et al., "High-energy chirped- and divided-pulse Sagnac femtosecond fiber amplifier," Opt. Lett., vol. 40, pp. 89-92, Jan. 2015.

[6] M. Kienel et al., "Analysis of passively combined divided-pulse amplification as an energy-scaling concept," Opt. Express, vol. 21, no. 23, pp. 29031-29041, Nov. 2013.

[7] A. Klenke, E. Seise, J. Limpert, and A. Tünnermann, "Basic considerations on coherent combining of ultrashort laser pulses," Opt. Express, vol. 19, no. 25, pp. 25379-25387, Dec. 2011.

[8] F. Guichard, L. Lavenu, M. Hanna, Y. Zaouter, and P. Georges, "Coherent combining efficiency in strongly saturated divided-pulse amplification systems," Opt. Express, vol. 24, no. 22, pp. 25329-2533, Oct. 2016.

[9] Y. Feng, B. M. Zhang, and J. Nilsson, "Photodarkening-induced phase distortions and their effects in single-channel and coherently combined Yb-doped fiber chirped pulse amplification systems," J. Lightwave Technol., vol. 36, no. 23, pp. 5521-5527, Dec. 2018.

[10] S. Palese, et al., "Coherent combining of pulsed fiber amplifiers in the nonlinear chirp regime with intra-pulse phase control," Opt. Express, vol. 20, no. 7, pp. 7422-7435, Mar. 2012.

[11] Y. Feng, H. Lin, and J. Nilsson, "Single-shot phase measurement and fluctuation analysis of Yb-doped fiber amplifier for nanosecond pulses," in Proc. CLEO, San Jose, CA, USA, 2018, Paper. SM2K.7.

[12] A. Brignon, "Collective Techniques for Coherent Beam Combining of Fiber Amplifiers", in Coherent Laser Beam Combining, Germany: Wiley, 2013, pp. 145-146.

[13] L. G. Kazovsky, et al., "All-fiber $90^{\circ}$ optical hybrid for coherent communications," Appl. Opt., vol. 26, no. 3, pp. 437-439, Feb. 1987.

[14] J. Ruppe, et al., "Multiplexed Coherent Pulse Stacking of 27 Pulses in a 4+1 GTI Resonator Sequence," in Proc. Lasers Congr. (ASSL, LSC, LAC), Nagoya, Japan, 2016, Paper. AM4A.6.

[15] I. Astrauskas, et al., "High-energy pulse stacking via regenerative pulseburst amplification," Opt. Lett., vol. 42, no. 11, pp. 2201-2204, Jun. 2017.

[16] G. D. Goodno, C. Shih, and J. E. Rothenberg, "Perturbative analysis of coherent combining efficiency with mismatched lasers," Opt. Express, vol. 18 , no. 24 , pp. $25403-25414$, Nov. 2010.

[17] C. H. Henry, "Theory of the linewidth of semiconductor lasers," IEEE J. Quantum Electron., vol. 18, no. 2, pp. 259-264, Feb. 1982. 